Preprint INR-TH-2020-028

\title{
The mass-gap in Quantum Chromodynamics and a restriction on gluon masses
}

\author{
S.A. Larin \\ Institute for Nuclear Research of the Russian Academy of Sciences, Theory \\ Division \\ 60th October Anniversary Prospect 7a, Moscow 117312, Russia
}

\begin{abstract}
We prove that it is necessary to introduce the non-zero gluon masses into the fundamental Lagrangian of Quantum Chromodynamics in order to describe the mass-gap in the reaction of electronpositron annihilation into hadrons. A new restriction on the gluon masses is obtained. The renormalized theory with non-zero Lagrangian gluon masses is constructed.
\end{abstract}

\section{Introduction}

Quantum Chromodynamics (QCD) is considered as the real theory of strong interactions since the famous discovery [1] of asymptotic freedom. The colour gauge symmetry of the Lagrangian ensures renormalizability and the possibility to use perturbation theory in calculations. The gauge bosons of QCD, the gluons, are made massless to have gauge invariance. It is not possible to make gluons massive via the famous Englert-Brout-Higgs mechanism of the spontaneous symmetry breaking [2] since coloured Higgs particles are forbidden by experiments. 
In the present paper we prove that it is necessary to introduce the non-zero gluon masses into the fundamental Lagrangian of QCD to describe the massgap in the reaction of electron-positron annihilation into hadrons. The massgap in this case means that below the first physical two-pion threshold the value of the total cross section of electron-positron annihilation into hadrons is zero and becomes non-zero above the two-pion threshold.

The concept of a gluon mass is often used in contemporary studies of QCD. However, while sometimes it is used as a parameter in effective models, it is not understood as a fundamental mass appearing at the level of the QCD Lagrangian but rather as a dynamically purely non-perturbatively created property, see works [3]-[6] which are alo confirmed by the lattice QCD calculations [7].

This sets those studies aside from the present one where the gluon masses are introduced on the level of the fundamental QCD Lagrangian.

Hence we get the theory with massive gluons which is the theory of the massive vector bosoms. This non-abelian Yang-Mills theory [8] with masses of the Proca type is known to be non-renormalizable.

Recently it was found that such a theory is in fact on mass-shell renormalizable [9]. On mass-shell renormalizability means that although the Green functions are non-renormalizable the physical S-matrix elements are renormalizable, see e.g. [10]. Thus one can have the consistent theory with massive gluons.

Introduction of gluon mass terms into the QCD Lagrangian by hands breaks gauge invariance and that is why is not acceptable. But it turns out to be possible to introduce gluon masses via the mechanism of the spontaneous symmetry breaking with subsequent removal of the coloured Higgs particles from the Lagrangian, preserving renormalizability and unitarity of the theory. Thus the mechanism of the spontaneous symmetry breaking plays in this case the role of the effective mathematical tool to get the consistent theory with gluon masses.

\section{A restriction on the gluon masses and the mass-gap.}

The standard QCD Lagrangian is

$$
\begin{gathered}
L_{Q C D}=-\frac{1}{4} F_{\mu \nu}^{a} F^{a \mu \nu}+i \bar{\psi}_{f} \gamma^{\mu}\left(\partial_{\mu}-i g A_{\mu}^{a} T^{a}\right) \psi_{f}-m_{f} \bar{\psi}_{f} \psi_{f} \\
-\frac{1}{\xi}\left(\partial^{\mu} A_{\mu}^{a}\right)^{2}+\partial^{\mu} \bar{c}^{a}\left(\partial_{\mu} c^{a}-g f^{a b c} c^{b} A_{\mu}^{c}\right)+\text { counterterms }
\end{gathered}
$$


Notations are usual. The summation over the quark (flavour) index $f=$ $u, . ., t$ is assumed. The general covariant gauge with the parameter $\xi$ is chosen. $m_{f}$ is the renormalized quark mass, $g$ is the renormalized strong coupling constant, $g^{2} /\left(16 \pi^{2}\right) \equiv a_{s}$.

It is known [11], that the covariant gauge in (1) does not fix the gauge ambiguity uniquely. Hence QCD with the Lagrangian (1) is not the complete theory of strong interaction. It should be considered only as the theory which correctly reproduces the perturbative expansion of the complete theory [10]. One can choose e.g. the Hamilton gauge which fixes the gauge ambiguity uniquely [10] to get the complete theory. But this gauge is not convenient for perturbative calculations.

To show the necessity of the non-zero Lagrangian gluon masses for the mass-gap and to obtain a new restriction on the gluon masses let us consider electron-positron annihilation into hadrons via the virtual $Z$ boson.

The quantity to be considered is the squared matrix element of the $Z$ boson decay into hadrons summed over all final hadron states. It is expressed in the usual way as the imaginary part of a correlator of weak neutral quark currents

$$
\begin{gathered}
\sum_{h}<0\left|J^{\mu}\right| h><h\left|J^{\nu}\right| 0>=2 \operatorname{Im} \Pi^{\mu \nu}, \\
\Pi^{\mu \nu}=i \int e^{i q x}<0\left|T\left(J^{\mu}(x) J^{\nu}(0)\right)\right| 0>.
\end{gathered}
$$

Here $J^{\mu}$ is the neutral quark current coupled to the $Z$ boson in the Standard Model.

The $Z$ boson decay into hadrons is completely described within the Standard $S U(3) \times S U(2) \times U(1)$ Model. For our purpose we will work in the leading order of the weak coupling constant and in all orders of the strong coupling constant. In other words one considers the Källen-Lehmann spectral representation [12] for the correlator of weak neutral quark currents (2) within QCD, see e.g. [13] for the case of electron-positron annihilation into hadrons via the virtual photon. It is worthwhile to note that the KällenLehmann spectral representation is one of the most rigorous statements of Quantum Field Theory.

The natrix element in eq.(2) must be zero below the two-pion threshold since there are no physical states below this threshold. But perturbative QCD does give non-zero contributions below the two-pion threshold as it will be shown. Our purpose will be to nullify these contributions.

One type of Feynman diagrams among the diagrams contributing to the correlator (2) is of special interest. These are the three-loop diagrams consisting of two quark loops connected by two gluon propagators, each quark loop has a $Z$ boson vertex. The diagram of this type was first calculated in 
[14]. The imaginary parts of these diagrams contain, in particular, Cutcosky cuts going only through two gluon propagators. These cuts give the contributions to the cross section of electron-positron annihilation which start from zero energy if gluons are massless. The contributions are of the order $a_{s}^{2}$ in the strong coupling constant. Of course there is an infinite series of such contributions (starting from zero energy) coming from diagrams of higher orders in $a_{s}$.

One considers for convenience the famous $R$-ratio instead of the total cross section of electron-positron annihilation into hadrons. This ratio is the total cross-section itself divided by the tree level cross section of electron-positron annihilation into the muon-antimuon pare. One gets the perturbative QCD (pQCD) contribution to the $R$-ratio

$$
R(s)_{p Q C D}=\rho_{\text {gluon }}(s)+\rho_{\text {quark }}(s) .
$$

Here $s$ is the squared momentum transfer of the process. $\rho_{\text {gluon }}(s)$ is the gluon contribution starting from zero $s$. It is produced by the Cutcosky cuts going via the gluon propagators only. $\rho_{\text {quark }}(s)$ is the quark contribution starting from the $u$-quark threshold $s=4 m_{u}^{2}$, where $m_{u}$ is the mass of the lightest $u$-quark. It comes from the Cutcosky cuts going through quark propagators.

Thus pQCD gives non-zero contributions to $R(s)$ starting from zero $s$. This contradicts to experiments which dictate that non-zero contributions to $R(s)$ start only from the first physical threshold, i.e. the two-pion threshold $s=4 m_{\pi}^{2}$, where $m_{\pi}$ is the pion mass. Hence one must somehow nullify the pQCD contributions in the energy interval $0<s<4 m_{\pi}^{2}$.

The first naive suggestion is that one should not trust the perturbation theory below the two-pion threshold since the perturbative series heavily diverges at low energies. But the perturbative series is well defined at any energy since its coefficients are rigorously calculable in renormalizable theory at any energy.

One could also suggest that perturbative contributions are cancelled by non-perturbative terms, i.e. by contributions of the type

$$
e^{-1 / a_{s}}=0 \cdot a_{s}+0 \cdot a_{s}^{2}+\ldots,
$$

which are invisible in the perturbative expansion at the point $a_{s}=0_{+}$. But the non-perturbative terms have completely different analytical structure as compared to the perturbative terms. Hence non-perturbative contributions can not exactly cancel perturbative series in the continuous interval $0<s<$ $4 m_{\pi}^{2}$.

We adopt here a constructive approach that the perturbation theory adequately reproduces the perturbative expansion of the exact solution of the 
complete theory. We could obtain this exact solution if we can do enough mathematics. If the perturbative expansion is non-zero below the two-pion threshold then the exact solution is also non-zero there. Thus the perturbative contribution should be exactly zero below the two-pion threshold.

Sometimes one objects that the perturbation theory predicts its own failure at the Landau pole, so what sense does it make to calculate beyond that. But the perturbation theory can not predict its own failure if QCD is a physical theory, just because it should mathematically strictly produce exact zero below the two-pion threshold.

Thus the only way to move the gluon contributions $\rho_{\text {gluon }}(s)$ above the two-pion threshold is to introduce the non-zero Lagrangian gluon masses. Since the corresponding Cutcosky cuts (crossing only gluon propagators) in the lowest order in $a_{s}$ go only via two gluon propagators, we get the restriction for the lightest gluon mass $\left(2 M_{g l}\right)^{2}>\left(2 m_{\pi}\right)^{2}$ or

$$
M_{g l}>m_{\pi}
$$

One can argue that this restriction is not really impactful since the dynamically generated gluon mass [3]-[6] is of the order of $500 \mathrm{MeV}$ which is essentially higher than the restriction (5). But we would like to underline that these two masses are absolutely different masses. From one side it is the dynamically purely non-perturbatively generated mass in the theory with zero Lagrangian gluon masses. From another side it is the perturbative mass introduced into the fundamenal QCD Lagrangian. And this is the central point of the paper that it is necessary to introduce the non-zero Lagrangian gluon masses to describe the mass-gap in electron-positron annihilation into hadrons. Otherwise perturbative QCD gives unphysical contributions below the two-pion threshold which are not cancelled by other, non-perturbative contributions.

The Lagrangian gluon masses depend on the renormalization point. So the question arises what kind of the gluon mass $M_{g l}$ should we choose in (5).

The natural choice is to take the perturbative pole mass. It arises as a pole of the complete perturbative gluon propagator which is obtained after summation of all loop propagator insertions. The perturbative pole mass is known to be a renormalization group invariant. It is the perturbative pole gluon mass which is taken in (5).

We would like to mention that in the previous work [13] we considered the process of electron-positron annihilation into hadrons via the photon. In that case the corresponding Cutcosky cuts (crossing only gluon propagators) in the lowest order in $a_{s}$ go via three gluon propagators. Hence we got there the restriction $M_{g l}>\frac{2}{3} m_{\pi}$. 
Later it was realized that it is more efficient to analyze electron-positron annihilation via the virtual Z-boson to obtain a new stronger restriction on gluon masses which is presented in eq.(5).

To produce the mass-gap in the energy interval $0<s<m_{\pi}^{2}$ one should move also quark thresholds above the two-pion threshold. This can be done by imposing the restriction $M_{u}>m_{\pi}$ on the perturbative pole mass of the lightest $u$-quark. One can argue that this is too strong restriction for the perturbative mass and it is more proper for the constituent mass. It is indeed an interesting point. But we are talking about the perturbative pole mass which in its turn is the perturbative asymptotic series. This series heavily diverges. There is the possibility that one can obey the necessary restriction on the perturbative pole $u$-quark mass if one succeeds in resummation of this series. Otherwise perturbative QCD would give unphysical contributions below the two-pion threshold which are not cancelled by other, non-perturbative contributions.

Thus we should modify the QCD Lagrangian by adding the gluon masses.

\section{Adding the gluon masses to the QCD La- grangian.}

To construct QCD with massive gluons we will follow the approach of [9]. It is presently the only known way to obtain renormalizable (or on massshell renormalizable) theory with massive gluons without coloured scalars. One should start with a renormalizable theory using the Englert-Brout-Higgs mechanism of the spontaneous symmetry breaking [2]. Hence one adds to the standard QCD Lagrangian (1) the scalar part:

$$
\begin{gathered}
L_{Q C D+\text { scalars }}=-\frac{1}{4} F_{\mu \nu}^{a} F^{a \mu \nu}+i \bar{\psi}_{f} \gamma^{\mu}\left(\partial_{\mu}-i g A_{\mu}^{a} T^{a}\right) \psi_{f}-m_{f} \bar{\psi}_{f} \psi_{f}+ \\
\left(D_{\mu} \Phi\right)^{+} D^{\mu} \Phi+\left(D_{\mu} \Sigma\right)^{+} D^{\mu} \Sigma-\lambda_{1}\left(\Phi^{+} \Phi-v_{1}^{2}\right)^{2}-\lambda_{2}\left(\Sigma^{+} \Sigma-v_{2}^{2}\right)^{2} \\
-\lambda_{3}\left(\Phi^{+} \Phi+\Sigma^{+} \Sigma-v_{1}^{2}-v_{2}^{2}\right)^{2}-\lambda_{4}\left(\Phi^{+} \Sigma\right)\left(\Sigma^{+} \Phi\right)+L_{g f}+L_{g c} \\
\text { +counterterms, }
\end{gathered}
$$

where $\Phi(x)$ and $\Sigma(x)$ are the triplets of scalar fields in the fundamental representation of the $S U(3)$ colour group. We add two triplets to generate masses for all eight gluons. If one adds only one triplet of scalar fields then only five gluons get masses.

$\lambda_{i}$ are coupling constants of scalar self interactions. $v_{i}$ are the vacuum parameters. $L_{g f}$ is the gauge fixing part in some renormalizable gauge and $L_{g c}$ is the gauge compensating part with the Faddeev-Popov ghost fields. 
One makes shifts of scalar fields to generate masses of gluons:

$$
\Phi(x)=\left(\begin{array}{l}
\phi_{1}(x)+i \phi_{2}(x)+v \\
\phi_{3}(x)+i \phi_{4}(x) \\
\phi_{5}(x)+i \phi_{6}(x)
\end{array}\right), \quad \Sigma(x)=\left(\begin{array}{l}
\sigma_{1}(x)+i \sigma_{2}(x) \\
\sigma_{3}(x)+i \sigma_{4}(x)+v \\
\sigma_{5}(x)+i \sigma_{6}(x)
\end{array}\right) .
$$

Here we take for simplicity $v_{1}=v_{2} \equiv v$. This variant of the shifts is chosen to avoid non-diagonal in $A_{\mu}^{a}$ terms in the quadratic form of the gluon fields. One gets four massive Higgs fields and eight Goldstone bosons.

We obtain the following mass terms for gluons

$$
\begin{gathered}
L_{M}=m_{g l}^{2}\left[\left(A^{1}\right)^{2}+\left(A^{2}\right)^{2}+\left(A^{3}\right)^{2}+\frac{1}{2}\left(A^{4}\right)^{2}+\right. \\
\left.\frac{1}{2}\left(A^{5}\right)^{2}+\frac{1}{2}\left(A^{6}\right)^{2}+\frac{1}{2}\left(A^{7}\right)^{2}+\frac{1}{3}\left(A^{8}\right)^{2}\right],
\end{gathered}
$$

here $m_{g l}^{2} \equiv g^{2} v^{2}$ is the gluon mass parameter.

The scalar fields as usual are divided into unphysical Goldstone bosons and 'physical' Higgs fields. Here are four Higgs particles and eight Goldstone bosons after the spontaneous symmetry breaking.

For renormalization of ultraviolet divergences it is convenient to use the Bogoliubov-Parasiuk-Hepp (BPH) subtraction scheme [15]. This scheme has a convenient property that counterterms of primitively divergent Feynman diagrams are truncated Taylor expansions of diagrams themselves at fixed values of external momenta. Thus counterterms of diagrams depending on some masses are also mass dependent. The subtractions of the BPH scheme in the case of the spontaneous symmetry breaking can be chosen consistently [16] with the Slavnov-Taylor identities [17],[18].

Let us consider only Green functions without external Higgs particles since we want to get rid of the Higgs fields from the Lagrangian.

In a renormalizable gauge one can remove all diagrams containing the Higgs propagators together with subtractions corresponding to these diagrams. These diagrams and subtractions are distinguishable due to their specific dependence on the Higgs masses. The remaining diagrams without the Higgs propagators stay renormalizable since they are not influenced by the removed subtractions depending on the Higgs masses.

It means that one can remove from the Lagrangian all terms with the Higgs fields and the corresponding counterterms with the specific dependence on the Higgs masses. As a result one gets renormalizable theory of massive gluons without Higgs fields. This theory is renormalizable since it is obtained from the original renormalizable theory (6). The adding of the quark fields is straightforward. 
Unitarity of the new theory can be established in the standard way [10] by the transition to the unitary gauge in the generating functional of Green functions.

The above derivation with the removal of the Higgs fields from the Lagrangian can be compared with the following case. Let us consider standard QCD with six quark flavours. One can remove all diagrams with the top quark propagators and their subtractions from all Green functions. At the Lagrangian level it means the removal of all terms with the top quark field and corresponding counterterms from the Lagrangian. Then one is left with the standard QCD Lagrangian with five quark flavours which is also renormalizable and unitary.

The Lagrangian of the obtained theory of massive gluons is quite involved since it contains eight gluons and eight Goldstone bosons plus ghosts. To make it simpler one can consider the unitary gauge instead of a renormalizable gauge. Then Goldstone bosons and ghosts disappear and one has only physical degrees of freedom in the lagrangian.

Renormalizability is violated in the unitary gauge but on mass-shell renormalizability is known to survive. It means that only the S-matrix elements are renormalizable. As a result one obtains the on mass-shell renormalizable massive Yang-Mills theory of the Proca type. Hence the Englert-Brout-Higgs mechanism is an efficient mathematical tool to derive on mass-shell renormalizability of the massive Yang-Mills theory of the Proca type. This is far from to be obvious in advance.

The resulting Lagrangian of QCD with massive gluons in the unitary gauge is

$$
\begin{aligned}
L_{\text {massive } Q C D}=L_{M} & -\frac{1}{4} F_{\mu \nu}^{a} F_{\mu \nu}^{a}+i \bar{\psi}_{f} \gamma_{\mu} D_{\mu} \psi_{f}-m_{f} \bar{\psi}_{f} \psi_{f} \\
& + \text { counterterms }
\end{aligned}
$$

where the expression for $L_{M}$ with gluon mass terms is given in (8).

Let us note that on mass-shell renormalizability does not mean that quarks and gluons appear as external particles of $S$-matrix elements. It means that the $S$-matrix elements with the physical external particles are finite.

The one-loop $\beta$-function in the massive QCD with the Lagrangian (9) was calculated in [13]. The calculation of the $\beta$-function is simpler of course in the minimal subtraction scheme where it does not depend on masses of the theory.

It turns out that asymptotic freedom remains valid in QCD with massive gluons. 


\section{Conclusions}

We have demonstrated that it is impossible to produce the mass-gap for the process of electron-positron annihilation into hadrons in QCD with zero Lgrangian gluon masses. One should introduce the non-zero Lagrangian gluon masses into the fundamental Lagrangian of QCD to produce the necessary mass-gap. We have obtained the new restriction on the lowest perturbative pole gluon mass $M_{g l}>m_{\pi}$.

We have also demonstrated that it is possible to construct QCD with non-zero Lagrangian gluon masses retaining renormalizability and unitarity of the theory.

\section{Acknowledgements}

The author is grateful to the collaborators of the Theory division of INR for helpful discussions. This research was supported by the Munich Institute for Astro- and Particle Physics (MIAPP) which is funded by the Deutsche Forschungsgemeinschaft (DFG, German Research Foundation) under Germany's Excellence Strategy EXC-2094-390783311.

\section{References}

[1] D.J. Gross, F. Wilczek, Phys. Rev. Lett. 30 (1973) 1343;

H.D. Politzer, Phys. Rev. Lett. 30 (1973) 1346.

G. 't Hooft, report at the Marseille Conference on Yang-Mills Fields, 1972 .

[2] P.W. Higgs, Phys. Lett. 12 (1964) 132.

F.Englert and R. Brout, Phys. Rev. Lett. 13 (1964) 321.

[3] J.M. Cornwall, Nucl. Phys. B 157 (1979) 392.

[4] J.M. Cornwall and A. Soni, Phys. Lett. 120 B (1983) 431.

[5] F.R. Graziani, Zeitschrift fr Physik 33 (1987)397.

[6] A. Aguilar and J. Papavassiliou, Eur. Phys. J. A 35 (2008) 189.

[7] UKQCD Collaboration, Phys. Rev. D 60 (1999) 094507.

[8] C.N. Yang and R.L. Mills, Phys. Rev. 96 (1954) 191.

[9] S.A. Larin, Phys.Part.Nucl. 44 (2013) 386; e-Print: hep-ph/0503198. 
[10] L.D. Faddeev and A.A. Slavnov. Gauge fields. Introduction to quantum theory, Front. Phys. 83 (1990) 1.

[11] V.N. Gribov, Nucl. Phys. B139 (1978) 1.

[12] G. Källen, Helv. Phys. Acta 25 (1952) 417; H. Lehmann, Nuovo Cim. 11 (1954) 342.

[13] S.A. Larin, AIP Conf.Proc. 1701 (2016) 1, 070003; e-Print: 1304.8107 [hep-ph].

[14] B.A. Kniehl and J.H. Kuehn, Nucl. Phys. B 329 (1990) 547.

[15] N.N. Bogoliubov and O.S. Parasiuk, Acta Math. 97 (1957) 227.

K. Hepp, Comm. Math. Phys. 2 (1966) 301.

[16] K. Symanzik, Comm. Math. Phys. 16 (1970) 48.

[17] A.A. Slavnov, Theor. Math. Phys. 10 (1972) 99.

[18] J.C. Taylor, Nucl. Phys. B 33 (1971) 436. 